coloproctology 2012 $\cdot 34: 113-113$

DOI 10.1007/s00053-012-0264-x

Online publiziert: 15. März 2012

๑) Springer-Verlag 2012

V.W. Fazio

Cleveland Clinic, Cleveland, OH, USA

\title{
Eingeladener Kommentar zu D. Geisler and T. Garrett
}

\section{Laparoskopische kolorektale Single-Incision- Chirurgie. Erfahrungen eines einzelnen Chirurgen in 102 konsekutiven Fällen}

Masse für die Evaluation zusammenkommen zu lassen. Also wäre das Beste, was man in diesem Stadium erreichen kann, eine Vergleichsstudie - und dies wird die Arbeit der Zukunft sein. Wie Dr. Geisler einräumt, liegen die Limitierungen dieser Studie woanders. Es ist kein Langzeitergebnis (z. B. Hernie an der Zugangsstelle) verfügbar und nicht einmal Kurzzeitergebnisse wie Schmerzkontrolle und Patientenzufriedenheit wurden verzeichnet. Ganz intuitiv würde man diese als mindestens vergleichbar zur konventionellen laparoskopischen kolorektalen Chirurgie erwarten, wenn nicht sogar als überlegen. Insgesamt glaube ich aber, dass dieser vorliegende Beitrag eine wertvolle Ergänzung im Diskurs über dieses Thema darstellt.

\section{Korrespondenzadresse}

\section{V.W. Fazio}

Cleveland Clinic

Cleveland, $\mathrm{OH}$, USA

faziov@ccf.org

von Technique in Coloproctology darlegt [2], um eine persönliche, unkontrollierte Patientenserie ohne klare definierte Einschluss- und Ausschlusskriterien. Allerdings traf dies für alle frühen Studien zur laparoskopischen kolorektalen Chirurgie zu. Im Fall der laparoskopischen Chirurgie bei kolorektalen Karzinomen brauchten große randomisierte kontrollierte Studien beinahe 10 Jahre, um eine kritische
Interessenkonflikt. Der Autor gibt an, dass kein Interessenkonflikt besteht.

\section{Literatur}

1. Geisler D, Garrett T (2011) Single incision laparoscopic colorectal surgery: a single surgeon experience of 102 consecutive cases. Tech Coloproctol. doi:10.1007/s10151-011-0756-7

2. Bergamaschi R (2011) More or LESS. Tech Coloproctol. doi:10.1007/s10151-011-0778-1 